\title{
CCSVI IN MULTIPLE SCLEROSIS: IS IT THE END?
}

\author{
Alessandro Rasman \\ University of Trieste, Italy
}

\author{
LETTER TO EDITOR \\ Phlebological Review 2017; 25, 1: 91-92 \\ DOl: https://doi.org/10.5114/pr.2017.72539 \\ Submitted: 3.12 .2017 \\ Accepted: 14.12 .2017 \\ ADDRESS FOR CORRESPONDENCE \\ Alessandro Rasman \\ University of Trieste, Italy \\ alessandro.rasman@libero.it
}

\section{DEAR EDITOR,}

Eight years ago, Paolo Zamboni, an Italian vascular surgeon, created a firestorm in the MS community with his hypothesis that MS, believed to be an autoimmune disease, could have a vascular connection. His research has shown that patients with MS had restricted venous flow from the brain and spinal cord, a condition he named chronic cerebrospinal venous insufficiency (CCSVI). His small observational study (65 patients assessed) demonstrated that PTA of venous strictures in patients with CCSVI is safe and, especially in patients with relapsing-remitting course of the disease, positively influenced the clinical status and quality of life of MS patients [2].

Zamboni's theory propelled wide-ranging research and the creation of a multidisciplinary society: the International Society for Neurovascular Disease to promote research on neurovascular pathologies. However, a study conducted by neurologists did not demonstrate association of CCSVI with MS [5].

MS neurologists, official gatekeepers of the disease, dismissed and even mocked Zamboni's theory and the scientific integrity of his research. Nonetheless, thousands of Italians with MS (the incidence of MS in Italy is high) spurred by anecdotal reports of improvements spread on social media, sought such a treatment. Many patients with MS who did not improve after standard pharmacological treatment or presented with contraindications for such a therapy, were at least interested in relieving symptoms of MS or improving quality of life. They also demanded that venoplasty for MS should be covered by the Italian healthcare system, as it is for other vascular pathologies. Reported responses to CCSVI treatment were as varied as the MS population itself - some patients experienced lasting benefits in terms of renewed energy, better balance, and improved bladder control [3]. In others, early improvements vanished with time, while some patients did not improve, or even experienced complications. There were three fatalities associated with this treatment reported in the literature [4]

MS patients called for a randomised double-blinded placebo-controlled trial performed in a similar manner to those aimed at testing new drugs. Recently a long-awaited Italian clinical trial investigating the safety and efficacy of venous percutaneous transluminal angioplasty (PTA) for the treatment of patients with multiple sclerosis (MS) has been published. Zamboni was principal investigator and senior author of this study, named "Brave Dreams" (Brain Drainage Exploited Against Multiple Sclerosis) [1]. The study wanted to provide an answer regarding the efficacy of PTA in terms of patients' functional disability (balance, motor, sensory, visual, and bladder function, cognitive, and emotional status), which are meaningful clinical outcomes but not directly associated with inflammatory lesions detected by imaging studies. In fact, an important part of patients' expectations, sustained and amplified by anecdotal data, has to do precisely with these functional aspects. The study confirmed a high prevalence (74\%) of CCSVI in patients with MS. It also found various and complex types of venous malformations, including closed jugular valves. PTA was effective in terms of jugular blood flow restoration only in 53\% of patients. According to the authors of this paper, PTA was found to be safe but clinically largely ineffective. PTA did not result in functional improvements (such as improvements in gait, balance, bladder control, fatigue, etc.) and did not reduce the mean number of new combined brain lesions detected by MRI at 12 months. Consequently, such a treatment should not be recommended in patients with MS.

However, anyone who has read this seven-page long report can find a second, somewhat contradictory conclusion - that MS patients benefited from improved cerebral blood flow after angioplasty in terms of some MRI-detected lesions. Seventy-seven per cent of patients in the treatment group, $22 \%$ more than in the sham group, were free of gadolinium-enhancing lesions at 12 months. The importance of this finding was explained in the study: 
"Gadolinium enhancement is a marker of damage to the blood-brain barrier, whose time course depends on lymphatic drainage and hence on venous drainage from the skull". According to the authors, this should be further analysed and investigated [1].

The paper was accompanied by an editorial written by three MS neurologists, scathing in its denouncement of CCSVI, CCSVI advocacy, and the role of social media in spreading ineffective medicine. The authors of this editorial declared that the study was rigorous and definitive, and its results unequivocal [6]. But is it really so?

The author declares no conflict of interest.

\section{References}

1. Zamboni P., Tesio L., Galimberti S., Massacesi L., Salvi F., D’Alessandro R., Cenni P., Galeotti R., Papini D., D’Amico R., Simi S., Valsecchi M.G., Filippini G.; Brave Dreams Research Group. Efficacy and safety of extracranial vein angioplasty in multiple sclerosis: a randomized clinical trial. JAMA Neurol 2017; doi: 10.1001/jamaneurol.2017.3825

2. Zamboni P., Galeotti R., Menegatti E., Malagoni A.M., Gianesini S., Bartolomei I., Mascoli F., Salvi F. A prospective open-label study of endovascular treatment of chronic cerebrospinal venous insufficiency. J Vasc Surg 2009; 50: 1348-58.e1-3.

3. Bavera P.M. May symptoms of chronic cerebrospinal venous insufficiency be improved by venous angioplasty? An independent 4-year follow up on 366 cases. Veins Lymphatics 2015; 4: 5400.

4. Dake M.D., Dantzker N., Bennett W.L., Cooke J.P. Endovascular correction of cerebrovenous anomalies in multiple sclerosis: A retrospective review of an uncontrolled case series. Vasc Med 2012; 17: 131-7.

5. Comi G., Battaglia M.A., Bertolotto A., Del Sette M., Ghezzi A., Malferrari G., Salvetti M., Sormani M.P., Tesio L., Stolz E., Zaratin P., Mancardi G.; CoSMoCollaborative Study Group. Observational case-control study of the prevalence of chronic cerebrospinal venous insufficiency in multiple sclerosis: results from the CoSMo study. Mult Scler 2013; 19: 1508-17.

6. Green A.J., Hooman K., Josephson S.A. Combating the spread of ineffective medical procedures: a lesson learned from multiple sclerosis. JAMA Neurol 2017; doi: 10.1001/jamaneurol.2017.3066 